AperTO - Archivio Istituzionale Open Access dell'Università di Torino

Use of intravenous immunoglobulin in patients with active vasculitis associated with concomitant infection

This is a pre print version of the following article:

Original Citation:

Availability:

This version is available http://hdl.handle.net/2318/1521200

since 2016-10-04T13:07:55Z

Published version:

DOI:10.1097/RHU.0000000000000201

Terms of use:

Open Access

Anyone can freely access the full text of works made available as "Open Access". Works made available under a Creative Commons license can be used according to the terms and conditions of said license. Use of all other works requires consent of the right holder (author or publisher) if not exempted from copyright protection by the applicable law. 
This is the author's final version of the contribution published as:

Simoes, Joana; Sciascia, Savino; Camara, Ines; Baldovino, Simone; Karim, Yousuf; Roccatello, Dario; Cuadrado, Maria Jose. Use of intravenous immunoglobulin in patients with active vasculitis associated with concomitant infection. JCR: JOURNAL OF CLINICAL RHEUMATOLOGY. 21 (1) pp: 35-37.

DOI: 10.1097/RHU.0000000000000201

The publisher's version is available at:

http://content.wkhealth.com/linkback/openurl?sid=WKPTLP:landingpage\&an=00124743-201501000-00009

When citing, please refer to the published version.

Link to this full text:

http://hdl.handle.net/2318/1521200 


\section{Use of Intravenous Immunoglobulin in Patients With Active Vasculitis Associated With Concomitant Infection}

Simoes, Joana $\mathrm{MD}^{*}$; Sciascia, Savino MD, $\mathrm{PhD}^{* \dagger}$; Camara, Ines $\mathrm{MD}^{*}$; Baldovino, Simone $\mathrm{MD}^{\dagger}$; Karim, Yousuf $\mathrm{MD}^{\ddagger}$; Roccatello, Dario $\mathrm{MD}^{\dagger}$; Cuadrado, Maria Jose MD, $\mathrm{PhD}^{\ddagger}$

The vasculitides are a heterogeneous group of diseases, comprising necrotizing and nonnecrotizing vessel diseases. Patients with vasculitis frequently develop infections, mainly as a consequence of treatments prescribed to treat their disease. Corticosteroids, immunosuppressants, and most immunomodulating agents (eg, anti-tumor necrosis factor or anti-CD20 monoclonal antibodies) facilitate infections, and combined therapies further increase that risk. ${ }^{1}$ In immunosuppressed patients, infections are sometimes life threatening and are one of the major causes of deaths. ${ }^{1}$

However, active vasculitis and infection can coexist, and treatment for these conditions is a challenge for physicians. Therefore, alternative therapeutic approaches rather than conventional immunosuppressants are needed to limit the disease activity when a concomitant infection is suspected.

Intravenous immunoglobulin (IVIG) has been used as a replacement therapy in patients with primary and secondary immunodeficiencies and in the treatment of many autoimmune and systemic inflammatory disorders. ${ }^{2}$ Moreover, their potential role against infections has been speculated. ${ }^{3}$ In the spectrum of systemic vasculitis, there is clear evidence of the benefit of IVIG in Kawasaki disease, and it is now also considered a therapeutic option in refractory antineutrophil cytoplasmic antibody-associated vasculitis. ${ }^{4} 5$ For other vasculitis, such as polyarteritis nodosa, Henoch-Schönlein purpura (HSP), or Behçet disease (BD), only a few case reports have described a beneficial effect of IVIG.

We report the largest cohort focusing on clinical effects of IVIG in patients with active vasculitis and concomitant infection.

\section{METHODS}

This observational, retrospective study included 14 patients with vasculitis and concomitant infection attending the Louise Coote Lupus Unit at St Thomas Hospital, London, or the Centro di Ricerche di Immunologia Clinica ed Immunopatologia e Documentazione su Malattie Rare, Università di Torino, Italy, from January 2001 to February 2012, who received at least 1 cycle of IVIG (400 mg/kg per day for 5 days).

The underlying vasculitic disorder was granulomatosis with polyangiitis (GPA) in 5 patients, 2 patients with Churg-Strauss syndrome, 2 patients with cutaneous vasculitis, and microscopic polyangiitis (MPA), HSP, $\mathrm{BD}$, pulmonary and systemic small-sized vasculitis were diagnosed in 1 patient, respectively. All the patients fulfilled the current classification criteria ${ }^{6}$ for the diagnosed vasculitis. All patients received highdose IVIG $(0.4 \mathrm{~g} / \mathrm{kg}$ per day) during 5 days on the grounds of a suspected or proven concomitant infection.

Intravenous immunoglobulin was added after immunosuppression had been discontinued. Patients were evaluated by a rheumatologist as an inpatient and during follow-up. The resolution of the acute episode of infection and concomitant active vasculitis causing the referral to our units constituted the endpoint. The outcome was classified as no response, partial remission, or complete remission according to the global physician assessment, improvement in disease activity according to clinical and analytical parameters.

The diagnosis of infection was based on the positive culture results of a pathogenic microorganism. For those with negative results of microorganism culture, infection was diagnosed by typical symptoms, signs, imaging investigations, and laboratory evaluations, combined with positive response to the standard 
antibacterial therapy. In patients who achieved complete or partial remission, immunosuppressant therapy was reintroduced or added after infection subsided.

\section{RESULTS}

The results are summarized in the Table 1 . All but 1 patient (patient 5 ) had received immunosuppression and/or intravenous (IV) corticosteroids prior to treatment with IVIG. Patient 5 was admitted with a first presentation of MPA and concomitantly developed hospital-acquired pneumonia.

Seven patients (50\%) achieved complete remission (3 GPA, 2 Churg-Strauss syndrome, 1 pulmonary vasculitis, and 1 cutaneous vasculitis); 3 (21\%) achieved partial remission (1 HSP, 1 MPA, 1 systemic vasculitis); and 4 (29\%) did not respond (2 GPA, 1 BD, 1 cutaneous vasculitis).

Among the patients with complete or partial response (71\%), the time free of relapse during the 24month follow-up was $20.9 \pm 8.5$ months.

Three patients died: patient 7 with acute interstitial pneumonia and patients 6 and 10 with sepsis. No adverse effects of IVIG were reported. An infectious agent was identified in all patients (Table 1) except in patient 11, admitted because of severe hemoptysis, shortness of breath, and fever. His blood test showed elevated markers of inflammation and leukocytosis. A computed tomography scan of the chest reported multifocal consolidations.

All the patients were treated with concomitant antibiotic therapy based on the results of microorganism culture.

\section{DISCUSSION}

Intravenous immunoglobulin has long been used as a rescue medication in autoimmune conditions. ${ }^{7-}$ ${ }^{9}$ However, available data on the use of IVIG in vasculitis and concomitant infection rely mainly on a large number of case reports. Vasculitis can be life threatening, and infections are one of the major causes of death. It has been reported in a prospective study, among the observed 85 of 278 fatalities, 11 patients (12.4\%) died of infections ( 5 of bacterial pneumonia and 6 with septicemia). ${ }^{10}$ In another study on the causes of early vasculitis-associated deaths, 13 of 60 fatalities during the first year after diagnosis were attributed to infections. ${ }^{11}$

This observational, retrospective, chart- and database-driven, 2-center clinical study aimed to evaluate the beneficial effects and safety profile of high-dose IVIG in the largest series of vasculitis patients with concomitant infections. The clinical features presented in our cohort at the time of IVIG administration included cutaneous, hematological, and lower and upper respiratory tract. Our study emphasizes that treatment with IVIG is effective, with more than $70 \%$ of the patients experiencing any benefit, with total or partial remission.

Recently, in a meta-analysis about the use of IVIG in GPA, it has been proposed that the mechanisms of action of IVIG may involve both anti-inflammatory and antimicrobial properties. ${ }^{7-9,12-15}$ Based on this assumption, we have hypothesized that IVIG may be beneficial in patients with a flare of a primary vasculitis and concomitant severe infection, particularly useful when the temporary discontinuation of immunosuppressive therapy is required.

However, IVIG was not used specifically to treat infections or sepsis, as it has not been proven to be effective for these indications, ${ }^{16,17}$ but it was considered the safest option to treat vasculitis activity because immunosuppressive therapies could lead to a worsening of the infection. In these patients, the 
beneficial effects of IVIG are most probably multifactorial, acting through complement deactivation, receptor blockade, anti-idiotypes, and modulation of cytokine production. Treatment with IVIG was aimed to treat the activity of the disease without producing severe immunosuppression, whereas the concomitant antibiotic therapy would aim to improve the infection. Our experience is consistent with the reported major advantage of the use of IVIG in vasculitis, as it seems also to prevent infections due to the B-cell deficit induced by combined steroids and cytotoxic agents. ${ }^{1}$

In this study, IVIGs were administered at the dose of $0.4 \mathrm{~g} / \mathrm{kg}$ per day during 5 days based on our previous experience in autoimmune diseases other than vasculitides. ${ }^{18}$ We acknowledge that other schemes (2 $\mathrm{g} / \mathrm{kg}$ administered over either 2 or 5 consecutive days) have been used to treat rheumatic diseases. ${ }^{9}$ The difference between these 2 regimens has not been fully investigated.

We acknowledge that the interpretation of our results has to be taken with caution because the series is heterogeneous regarding the type of vasculitis and infections.

Nevertheless, the favorable outcome of 10 patients is encouraging, particularly because in our series IVIG was added only when the clinical condition of the patient had deteriorated, and it was sensed that antibiotic treatment might not be sufficient. Three patients died despite IVIG, as anecdotally reported in the literature, especially when active disease is associated with viral infection. ${ }^{19}$

In conclusion, in this cohort, IVIG was found to be effective in selected cases of vasculitis, when other therapeutic approaches (mainly immunosuppressors) were not appropriate, such as in patients with active disease and concomitant infection. Considering costs and limited availability of IVIGs treatment, we would recommend that randomized controlled trials are needed to support these observations.

\section{REFERENCES}

1. Guillevin L. Infections in vasculitis. Best Pract Res Clin Rheumatol. 2013; 27: 19-31.

2. Kazatchkine MD, Kaveri SV. Immunomodulation of autoimmune and inflammatory diseases with intravenous immune globulin. N Engl J Med. 2001; 345: 747-755.

3. Bayry J, Lacroix-Desmazes S, Kazatchkine MD, Kaveri SV. Intravenous immunoglobulin for infectious diseases: back to the pre-antibiotic and passive prophylaxis era? Trends Pharmacol Sci. 2004; 25: 306-310.

4. Martinez V, Cohen P, Pagnoux C, et al. Intravenous immunoglobulins for relapses of systemic vasculitides associated with antineutrophil cytoplasmic autoantibodies: results of a multicenter, prospective, openlabel study of twenty-two patients. Arthritis Rheum. 2008; 58: 308-317

5. Svetlicky N, Blank M, Zandman-Goddard G. The beneficial effects of intravenous immunoglobulin for antineutrophil cytoplasmic antibody-positive vasculitis. Isr Med Assoc J. 2012; 14: 568-569

6. Jennette JC, Falk RJ, Andrassy K, et al. Nomenclature of systemic vasculitides. Proposal of an international consensus conference. Arthritis Rheum. 1994; 37: 187-192.

7. Rogosnitzky M, Danks R, Holt D. Intravenous immunoglobulin for the treatment of Crohn's disease.Autoimmun Rev. 2012; 12: 275-280.

8. Marie I, Mouthon L. Therapy of polymyositis and dermatomyositis. Autoimmun Rev. 2011; 11: 6-13.

9. Bayry J, Negi VS, Kaveri SV. Intravenous immunoglobulin therapy in rheumatic diseases. Nat Rev 
10. Gayraud M, Guillevin L, le Toumelin P, et al. Long-term followup of polyarteritis nodosa, microscopic polyangiitis, and Churg-Strauss syndrome: analysis of four prospective trials including 278 patients. Arthritis Rheum. 2001; 44: 666-675.

11. Bourgarit A, Le Toumelin P, Pagnoux C, et al. Deaths occurring during the first year after treatment onset for polyarteritis nodosa, microscopic polyangiitis, and Churg-Strauss syndrome: a retrospective analysis of causes and factors predictive of mortality based on 595 patients. Medicine. 2005; 84: 323-330.

12. Cousens LP, Tassone R, Mazer BD, et al. Tregitope update: mechanism of action parallels IVIG. Autoimmun Rev. 2013; 12: 436-443.

13. Kaveri SV. Intravenous immunoglobulin: exploiting the potential of natural antibodies. Autoimmun Rev. 2012; 11: 792-794

14. Leardini G, Rigon C. The impact of the profile of biologics on treatment costs. Autoimmun Rev. 2013; 12: 842-847.

15. Maddur MS, Kaveri SV, Bayry J. Comparison of different IVIG preparations on IL-17 production by human $\mathrm{T}_{\mathrm{H}} 17$ cells. Autoimmun Rev. 2011; 10: 809-810.

16. Lacy JB, Ohlsson A. Administration of intravenous immunoglobulins for prophylaxis or treatment of infection in preterm infants: meta-analyses. Arch Dis Child Fetal Neonatal Ed. 1995; 72: F151-F155.

17. Pildal J, Gotzsche PC. Polyclonal immunoglobulin for treatment of bacterial sepsis: a systematic review. Clin Infect Dis. 2004; 39: 38-46.

18. Camara I, Sciascia S, Simoes J, et al. Treatment with intravenous immunoglobulins in systemic lupus erythematosus: a series of 52 patients from a single centre [published online ahead of print September 12, 2013]. Clin Exp Rheumatol. 2013.

19. Aries PM, Ullrich S, Gross WL. A case of destructive Wegener's granulomatosis complicated by cytomegalovirus infection. Nat Clin Pract Rheumatol. 2006; 2: 511-515 
TABLE 1 Demographic and Clinical Characteristics of the Patients Treated With IVIG

\begin{tabular}{|l|l|}
\hline Mechanism & Agent \\
\hline Targeting surface molecules on B cells & Ocrelizumab (fully humanized anti-CD20) \\
\hline \multirow{2}{*}{ Targeting B cell growth and survival factors } & Epratuzumab (fully humanized anti-CD22) \\
\hline & Atacicept \\
\hline & Blisibimod \\
\hline Toleragen molecule & Tabalumab \\
\hline Proteasome inhibition & Abetimus sodium \\
\hline Targeting co-stimulatory molecules & Bortezomib \\
\hline Targeting T cells & AMG 557 (against B7RP-1, an inducible co-stimulator ligand) \\
\hline Targeting cytokines - IL 6 & Edratide \\
\hline Targeting cytokines - type I interferons & Rigerimod \\
\hline & Laquinimod \\
\hline & Tocilizumab \\
\hline & Sirukumab \\
\hline
\end{tabular}

